\title{
Research on Temperature Control System Based on Three-Dimensional Fuzzy-Control
}

\author{
ZHANG Bin ${ }^{1, a}$ \\ 'Zaozhuang Science and Technology Vocational School, China \\ a1142169699@qq.com
}

Keywords: Three-dimensional fuzzy-control; control system; MATLAB.

Abstract. A temperature control system based on three-dimensional fuzzy-control is therefore proposed to cope with the problems of time-variation, nonlinear and uncertainty about underground environmental parameter. The precisely controlled variables are three-dimensional falsification, the design of the control system is determined and the hardware and software design of the control system is completed according to the decision of the fuzzy inference and the output information. The feasibility of the design is verified by MATLAB simulation and experimental test and the result shows that the convergence and the control efficiency of the temperature control based on the three-dimensional fuzzy algorithm are higher, and the overall mean variance can be controlled within $2^{\circ} \mathrm{C}$.

\section{Introduction}

Internal temperature control of mine has a very important influence on coal mine safety production. When the temperature reaches a certain value, it will lead to the oxidation of the coal seam, and even lead to accidents such as gas combustion, therefore, the strict and intelligent control underground temperature control is essential [1]. Due to work conditions and environmental parameters in a coal mine are time-variation, nonlinear and uncertainty, the conventional temperature control method and theory are difficult to guarantee the stability and safety of the temperature control. In recent years, with the development of computer technology, relying on its powerful adaptive ability and good control effect, fuzzy control theory has been widely recognized and applied, and it has superior control ability especially under the complex external conditions [2]. The intelligent temperature control can be completed after its design of hardware and software is finished. Finally, the feasibility of the algorithm is validated through simulation and experimental method.

\section{Analysis on three-dimensional fuzzy-control}

Algorithm Control Method. Fuzzy control algorithm that based on computer technology has a strong and efficient data processing capacity which is complex and nonlinear, due to the operation process of the controlled object does not require accurate mathematical model, and the control efficiency of multi variable parameters is higher [3]. According to the characteristics of temperature control environment in mine, the control scheme of the algorithm is established as shown in Fig.1.

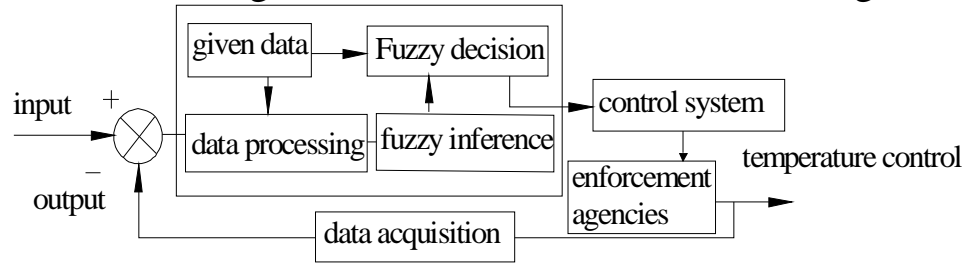

Fig.1 Fuzzy algorithm control scheme

The control part in temperature control system dominates the actuator (ventilation machine, flake ice machine, etc.). According to the final fuzzy data, the temperature control results are feedback to the output of the controller through the information collection system. A complete fuzzy control system requires four factors: the input/output variables of controller, fuzzy strategy, fuzzy rules and fuzzy reasoning [4]. 
Fuzzy Designing Rules. The main function of the fuzzy rules is to draw a lot of fuzzy conditions by using the manual control strategy, which is the key part of the fuzzy control. There are three inputs and one output in this paper, three inputs are respectively refers to the difference $(e)$ between the temperature before and the preset one, the change rate of the difference $(e c)$ between the temperature before and the preset one, the difference $(w)$ between the temperature controlled and the preset one. One output refers to temperature control $u$ of the implementation. Assuming the input is xi, the output is yi, and the design of fuzzy rule flow chart is shown in Fig.2.

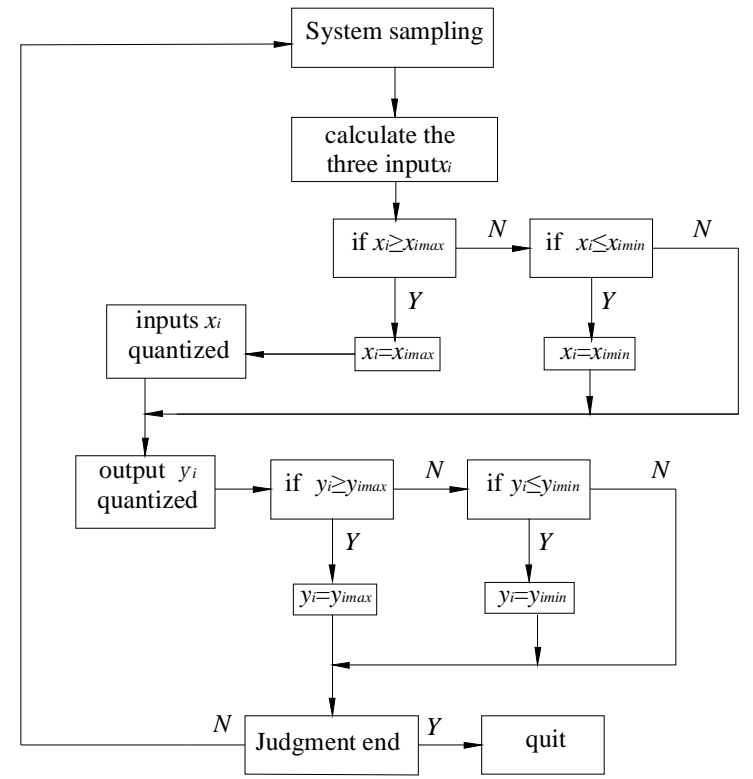

Fig.2 Flow chart of fuzzy rule

\section{System hardware design}

General Frame Design. According to the fuzzy control plan and the requirement of the field environment, the frame design of the hardware system is shown in Fig.3. When the temperature control system is working well, monitoring points of real-time data can be transferred to the PC side which can control the hardware system based on the tool kit of MATLAB, and then through the relevant instructions to control it fully.

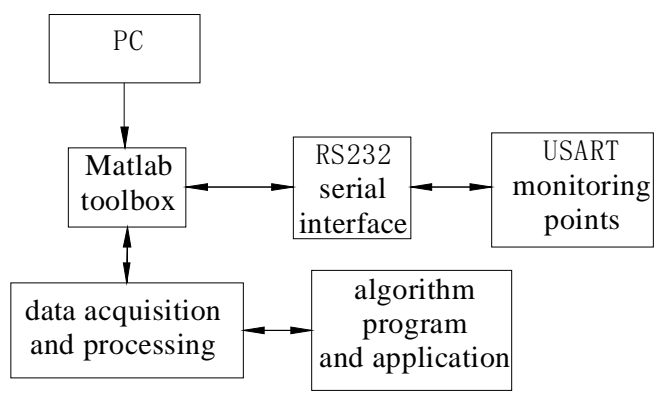

Fig.3 Hardware system framework

Hardware Module Design. The STC12C5A60S2 single chip microprocessor which has the characters of high-speed processing, anti-jamming and be suitable for the mine is selected in this paper. The microprocessor integrated MAX810 reset circuit, high speed DAC and 2 pulse width modulation, the interface circuit is shown in Fig.4.

Data Monitoring Module. The temperature and humidity sensor of SHT is selected in this paper, the sensor integrated serial port and DAC with high speed response, the detection error of the temperature is \pm 0.4 which means real time data monitoring can be realized accurately [5].

Power Module. The power module of the temperature control system design should consider the function of frequency compensation and voltage conversion. In this paper, the LM2596 chip is selected as the controller in power module. The chip can realize the function of power off and limit current. 
Relay Control Module. In order to control the on-off between the external device and the power module, the SRD relay is adopted to control the input/output interface and the external equipment through the switching signal. When the system works normally, the input/output of the microcontroller maintains a low level and all devices are activated under the control of the relay [6].

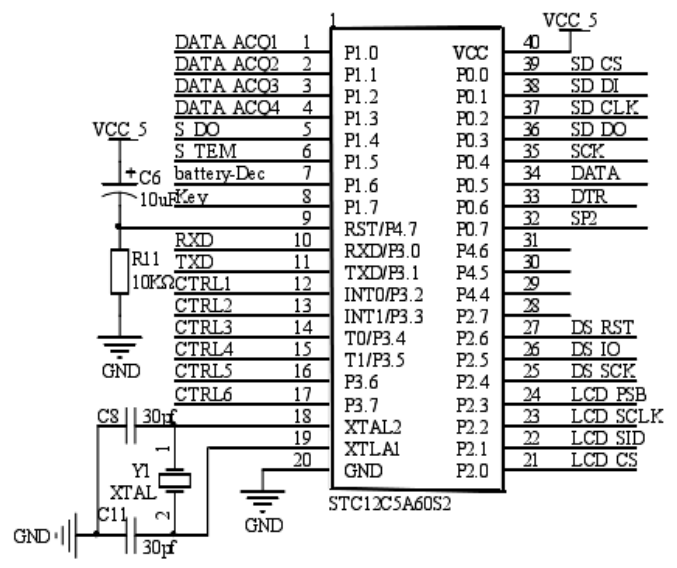

Fig.4 Single chip wiring diagram

\section{System software design}

The Overall Design Scheme. For the software design of the temperature control system, the overall design scheme is: time period of monitoring point collection can be set to 10 minutes, the temperature data will be uploaded to the PC through the serial port, and the PC port will output the control command automatically through MATLAB toolbox. In order to avoid data loss that caused by emergencies related, the EEPROM will preserve data when the power is off. The control process of the software system is shown in Fig.5, serial communication is adopted in the process of information transmission, the monitoring point sends out a signal and $\mathrm{PC}$ receiving and processing it to control the temperature, if the signal transmission is not be received, the pc will wait for the sampling period until the signal is comply be received.

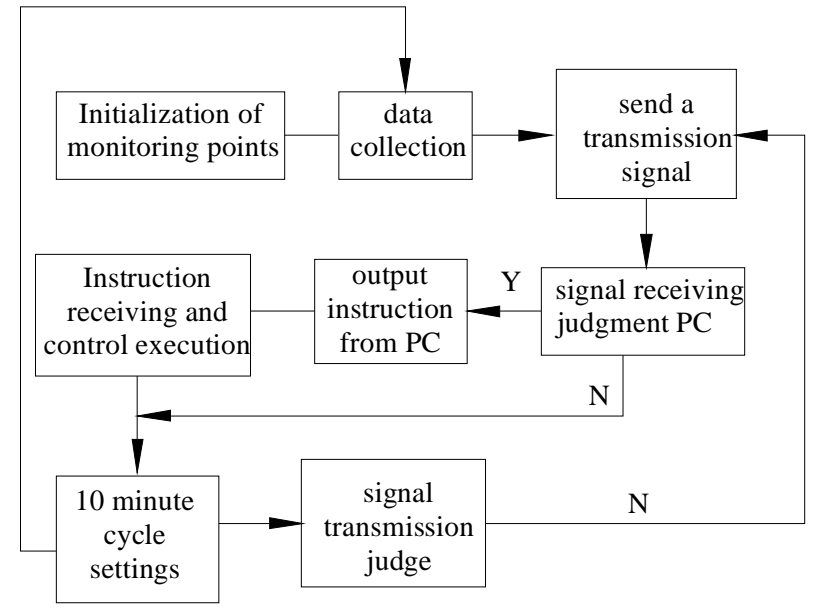

Fig.5 Flow chart of software control

Timing Control Program Design. Timing control is an important control method in the temperature control. The calculation formula of the timing initial value $T$ under the control of the 12 frequency division time is shown as Eq.1:

$$
T=\left(2^{n}-x\right) \cdot \frac{12}{f_{c}}
$$

In Eq. $1, n$ is the bit count of the counter, $x$ denotes the initial value for the count, $f_{c}$ is the clock pulse. In the timing terminal program, the timing value and the data acquisition period of the monitoring points are designed to be consistent and maintain the coordination between software and hardware. 
Design of Communication Program. Serial communication of data is based on MATLAB control, when the serial port event is interrupted, the function of instrcallback () which is equivalent to a subroutine control can be called in MATLAB. In this paper, the serial communication program is mainly divided into two steps. Fist, establish the main program and initialize related functions. Second, edit callback function and add the temperature control code to the function. In the processing of the callback function, it is required to guarantee the real-time performance of the temperature data and the three input parameters, fuzz up parameters by the algorithm and waiting for the next interrupt when the serial output signals.

\section{Conclusions}

A temperature control system is designed and studied based on the three-dimensional fuzzy-control method in this paper to cope with complex and changeable temperature underground. The study result shows that the three-dimensional fuzzy-control algorithm has a good control effect on the complex and nonlinear system with a broad application prospects. Under the guidance of the control algorithm in this paper, the design of temperature control system on hardware and software are finished which shows a clear goal. Finally, the advantages of fuzzy-control system can be seen according to the comprehensive comparison between MATLAB and experimental test. The control system has an important significance on improving the safety of coal mine production, and at the same time, it provides some guidance for improving the control ability of other systems.

\section{References}

[1] X.R. JIANG: Coal Mine Machinery,Vol(2015)No.36,p5-8..

[2] Y.SH. MA: Journal of Hebei University of Science \& Technology,Vol(2010) No24,p20-23.

[3] Y.Q. HU: Proceedings of the CSEE,Vol(2003)No23,p137-140.

[4] B.LI: Mechanical Engineering \& Automation,Vol(2015)No4,p78-81.

[5] Sh.R.Wang, Ch.H.Yao: Chinese Foreign Language, Vol(2013)No.5,p4-10.

[6] R. Kai: Journal of Nanjing Normal University, Vol(2009) No9,p18-21. 